\title{
11 PERCEÇÕES, ATITUDES E EMOÇÕES DOS DOENTES SUBMETIDOS A INTERNAMENTO PSIQUIÁTRICO COMPULSIVO: REVISÃO INTEGRATIVA
}

\author{
| Tânia Sofia Pereira Correia ${ }^{1} \mid$
}

\section{RESUMO}

CONTEXTO: O internamento compulsivo em Psiquiatria é um dos temas mais desafiantes desta área da saúde devido às questões éticas envolvidas. A experiência de estar sob cuidados involuntários, nomeadamente em internamento compulsivo, caracteriza-se por sentimentos negativos face à perda de liberdade e autonomia.

OBJETIVOS: Conhecer perceções, atitudes e emoções dos doentes submetidos a internamento compulsivo.

MÉTODOS: Revisão integrativa da literatura através de pesquisa nas bases de dados EBSCO e Trip Database nas duas primeiras semanas de janeiro de 2018, cujos critérios de inclusão foram: estarem disponíveis em full text, de abordagem quantitativa, qualitativa e/ou revisões de literatura, nos idiomas Português, Inglês e Espanhol e terem como tema de pesquisa as perceções, atitudes e emoções de doentes internados compulsivamente em psiquiatria.

RESULTADOS: Foram encontrados 8 artigos que dão resposta ao objetivo do estudo, incluem uma revisão de literatura, quatro estudos observacionais, um ensaio controlado, um estudo qualitativo e um estudo de validação de escala.

CONCLUSÕES: Face à experiência de internamento compulsivo, foram identificadas perceções, atitudes e emoções maioritariamente negativas, mas também positivas e ambivalentes. Conclui-se que estas vivências podem ter consequências para além do internamento e que há práticas que devem ser aperfeiçoadas no sentido de melhorar a experiência do doente sujeito a internamento compulsivo.

PALAVRAS-CHAVE: Internação compulsória de doente mental; Coerção; Satisfação do paciente; Emoções

\section{RESUMEN}

"Percepciones, actitudes y emociones de los enfermos sometidos a ingreso compulsivo: Revisión integrativa"

CONTEXTO: El ingreso compulsivo en Psiquiatría es uno de los temas más desafiantes de esta área de salud debido a las cuestiones éticas envueltas. La experiencia de estar bajo cuidados involuntarios, en un régimen de ingreso compulsivo, se caracteriza por sentimientos negativos en relación a la pérdida de libertad y autonomía.

OBJETIVOS: Conocer las percepciones, actitudes y emociones de los enfermos sometidos al ingreso compulsivo.

MÉTODOS: Revisión integrativa de la literatura a través de la búsqueda en las bases de datos EBSCO eTrip Database, en las dos primeras semanas de enero de 2018, cuyos criterios de inclusión fueron: estar disponibles en texto completo, cuantitativa, cualitativa y / o revisiones de la literatura, en portugués, inglés y español y tienen como tema de investigación de las percepciones, actitudes y emociones de pacientes internados compulsivamente en psiquiatría.

RESULTADOS: Se encontraron ocho artículos que dan respuesta al objetivo del estudio, incluyen una revisión de literatura, cuatro estudios observacionales, un ensayo controlado, un estudio cualitativo y un estudio de validación de escala.

CONCLUSIONES: Frente a la experiencia de internamiento compulsivo, se identificaron percepciones, actitudes y emociones mayoritariamente negativas, pero también, positivas y ambivalentes. Se concluye que estas vivencias pueden tener consecuencias más allá del internamiento y que hay prácticas que deben ser perfeccionadas en el sentido de mejorar la experiencia del paciente sujeto a internamiento compulsivo.

DESCRIPTORES: Internación compulsoria del enfermo mental; Coerción; Satisfacción del paciente; Emociones

\begin{abstract}
"Perceptions, attitudes and emotions of the patients subjected to involuntary psychiatric hospitalization: Integrative review"

BACKGROUND: Involuntary hospitalization in Psychiatry is one of the most challenging themes in this specialty due to the ethical issues involved. The experience of being under involuntary care, namely in compulsory hospitalization, is characterized by negative feelings in the face of loss of freedom and autonomy.

AIM: To investigate the perceptions, attitudes and emotions of the patients that are admitted into involuntary hospitalization.

METHODS: Integrative literature review through data search on the EBSCO database and on the Trip Database, in the first two weeks of January 2018, whose inclusion criteria were: to be available in full text, quantitative, qualitative and / or literature reviews, in the Portuguese, English and Spanish languages, attitudes and emotions of patients hospitalized compulsively in psychiatry.

RESULTS: We found eight articles that met the study's objective, including a literature review, four observational studies, a controlled trial, a qualitative study and a scale validation study.

CONCLUSIONS: Perceptions, attitudes and emotions that were mostly negative, but also positive and ambivalent, were identified in view of the experience of compulsive hospitalization. It is concluded that these experiences can have consequences beyond hospitalization and that there are practices that must be improved in order to improve the experience of the patient subject to compulsory hospitalization.
\end{abstract}

\section{KEYWORDS: Commitment of mentally ill; Coercion; Patient satisfaction; Emotions}

Submetido em 31-03-2018

Aceite em 16-07-2018

1 Mestre em Direção e Chefia dos Serviços de Enfermagem; Estudante na Escola Superior de Saúde Norte da Cruz Vermelha Portuguesa, curso de Pós-Licenciatura de Especialização em Enfermagem de Saúde Mental e Psiquiátrica; Enfermeira no Centro Hospitalar do Baixo Vouga, EPE, Pedopsiquiatria, Aveiro, Portugal, tsp.correia@gmail.com

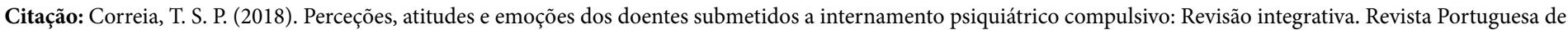
Enfermagem de Saúde Mental (20), 81-90. doi: 10.19131/rpesm.0230 


\section{INTRODUÇÃO}

O internamento compulsivo de doentes com transtorno mental, devido ao compromisso da liberdade e autonomia individual, é um dos temas mais controversos e tem vindo a gerar debates jurídicos e éticos há mais de 100 anos. Durante os séculos XIX e XX, surgiram, em todo o mundo, diferentes abordagens e regulamentações de medidas compulsivas influenciadas por tradições culturais ou legais (European Commission, 2002).

A implementação de medidas compulsivas nos cuidados de saúde mental deve respeitar e equilibrar três interesses/argumentos diferentes: os direitos humanos básicos das pessoas envolvidas, a segurança pública e a necessidade de tratamento adequado da pessoa em questão (European Commission, 2002).

Os fatores associados ao internamento compulsivo, a sua prevalência e a insatisfação dos doentes têm vindo a ser estudados e investigados internacionalmente, especialmente a nível Europeu (Gabriel, 2017). São conhecidos muitos dos fatores de risco para internamento compulsivo. No entanto, os resultados dos estudos neste âmbito são difíceis de comparar por falta de representatividade ou por outras deficiências metodológicas (European Commission, 2002). A evidência sugere que são fatores importantes para o risco de internamento involuntário os diagnósticos (esquizofrenia e outros transtornos psicóticos), a intensidade das doenças psiquiátricas, os antecedentes de internamento compulsivo e níveis mais baixos de funcionamento social (Zinkler \& Priebe, 2002; European Commission, 2002; Myklebust, Sørgaard, Røtvold, \& Wynn, 2012).

A prevalência do internamento compulsivo varia significativamente em toda a Europa. Uns dados são apresentados sob a forma de taxas (número anual de admissões compulsivas por 100.000 habitantes) e outros em percentagens (European Commission, 2002). Isto deve-se ao facto de os dados de fontes oficiais serem frequentemente fornecidos por relatórios nacionais de saúde, departamentos de saúde, ou gabinetes estatísticos que utilizam diferentes definições ou métodos utilizados para o cálculo. Desta forma, verifica-se uma falta de dados internacionalmente comparáveis e a falta de estudos metodologicamente sólidos (European Commission, 2002).

Zinkler e Priebe (2002) realizaram uma revisão sobre internamento compulsivo de pessoas com transtorno mental em que os resultados revelam grandes variações em diferentes países europeus.
$\mathrm{Na}$ década de 90 verificou-se um aumento dos internamentos compulsivos em países como a Inglaterra e a Áustria, a nível regional e nacional, no entanto os países com maiores taxas são a Finlândia e a Alemanha. Também se apurou que as taxas são superiores em zonas urbanas (Zinkler \& Priebe, 2002). Nesta revisão, concluíram que altas taxas de internamento compulsivo podem dever-se à valorização da segurança pública e condições de vida vulneráveis. Por outro lado, baixas taxas podem estar relacionadas com desenvolvimento de trabalho comunitário significativo, como é o caso de Itália (Zinkler \& Priebe, 2002). No relatório Compulsory Admission and Involuntary Treatment in the EU da Comissão Europeia (2002) também verificaram diferenças notáveis entre os estados membros, variando da mais baixa, com apenas 6 por 100.000 habitantes em Portugal, para a mais alta, com 218, na Finlândia; ou em percentagens de internamentos compulsivos sobre todos os episódios de internamento psiquiátrico anual, que varia entre 3,2\% em Portugal a 30\% na Suécia (valores do ano de 1999 e/ou 2000). Este relatório divulga que, durante a última década, as taxas de internamento compulsivo mantiveram um padrão ligeiramente homogéneo. Estes dados contrariam conclusões que indicam uma tendência internacional de crescimento relatado em outros trabalhos científicos. O mesmo relatório considera que o aumento do número total de internamentos compulsivos deve ser contextualizado com as alterações dos padrões de cuidados prestados e com redução do tempo médio do internamento em detrimento de readmissões mais frequentes (European Commission, 2002). Isto é, face aos cuidados prestados atualmente, internamentos tendencialmente mais curtos e readmissões mais frequentes não significam mais dias de internamento compulsivo.

Os critérios legais para o internamento compulsivo dos doentes mentais são globalmente similares nos países europeus quando se trata de pacientes em risco para si próprios ou para os outros (Zinkler \& Priebe, 2002). Os mesmos autores acreditam que esta prática parece ser mais influenciada pelas crenças e valores dos profissionais de saúde mental do que pelos requisitos legais (Zinkler \& Priebe, 2002). No entanto, verificou-se que as taxas de internamento compulsivo na Europa é menor nos países que estipulam a inclusão obrigatória de um advogado independente no procedimento. Por consequência, os países em que não há apoio jurídico independente obrigatório apresentam taxas significativamente maiores (European Commission, 2002). 
A primeira Lei de Saúde Mental em Portugal surgiu em 1963. Os programas de saúde mental em 1985 e 1989 incluíam medidas importantes para integrar cuidados de saúde mental em hospitais gerais e desenvolver a saúde mental da comunidade. Em 1992 a legislação determina a integração de todos os centros de saúde mental em hospitais gerais (Almeida \& Molodynski, 2016). Todavia, o investimento em serviços de natureza comunitária em saúde mental, iniciado na década de 80, foi interrompido por razões políticas no início da década de 90 (Almeida \& Molodynski, 2016).Tornou-se premente a necessidade de rever a lei da saúde mental em Portugal de forma a dar resposta a recomendações externas, nomeadamente das Nações Unidas e Organização Mundial de Saúde (OMS), sobre a prestação de cuidados de saúde ao nível da comunidade, reabilitação psicossocial em estruturas residenciais, centros de dia e unidades de reabilitação profissional integradas na comunidade e adaptadas ao grau de autonomia de cada um (Almeida \& Molodynski, 2016).

Como resultado desta influência, é criada em 24 de julho de 1998 a Lei 36/98 e em 5 de fevereiro de 1999 é criado o Decreto-lei n. 35/99 que regula a lei, inclui a base da política de saúde mental e descreve detalhadamente a organização dos serviços de saúde mental. A lei da saúde mental tem cobertura nacional e aplica-se para todos os cidadãos portugueses. Define a política, os princípios gerais da prestação de serviços de saúde mental e os princípios reguladores para o internamento compulsivo de pessoas com doença mental, bem como os seus direitos. Determina que os cuidados de saúde mental devem ocorrer em contexto essencialmente comunitário e num ambiente o menos restritivo possível (Almeida \& Molodynski, 2016). Em resposta, foram tomadas medidas importantes neste sentido. No entanto, a hospitalização continua a consumir a maior fatia dos recursos (83\%) (Caldas de Almeida, 2009).

De acordo com o artigo $5^{\circ}$ da Lei $36 / 98$, todos os utilizadores dos serviços de saúde mental têm como direitos: receber informações adequadas sobre seus direitos, o plano de tratamento proposto e os efeitos esperados; tratamento e proteção com base no respeito pela individualidade e dignidade; autonomia para aceitar ou recusar intervenções, exceto em casos de detenção obrigatória ou em situações de emergência em que a não intervenção representaria riscos verificáveis para a pessoa ou para outros; aceitar ou recusar participar em investigações, ensaios clínicos; condições dignas e adequadas em serviços hospitalares e residenciais: habitabilidade, higiene, alimentação, segurança, respeito e privacidade; receber apoio no exercício dos direitos de protesto e reclamação (Almeida \& Molodynski, 2016; Lei n. ${ }^{\circ}$ 36/98 - Lei de Saúde Mental, 1998).

De acordo com o artigo $12^{\circ}$ da Lei de Saúde Mental, os critérios para internamento compulsivo compreendem: pessoa que, em virtude de um transtorno mental grave, represente um perigo para si próprio ou para outros (bens jurídicos de relevante valor, próprios ou alheios, de natureza pessoal ou patrimonial); recusa de tratamento médico necessário; incapacidade para avaliar o significado e as implicações do consentimento e da ausência de tratamento que pode resultar em uma deterioração significativa da sua condição (Almeida \& Molodynski, 2016; Lei n. ${ }^{\circ}$ 36/98 - Lei de Saúde Mental, 1998).

$\mathrm{O}$ artigo $13^{\circ}$ refere que a detenção pode ser solicitada pelo representante legal de uma pessoa que sofre de transtorno mental, qualquer pessoa elegível para solicitar sua interdição, autoridades de saúde pública, Ministério Público, médicos ou o diretor clínico de uma instituição, nos casos em que o conhecimento de transtorno mental acontece durante admissão voluntária naquela instituição (Caldas de Almeida, 2009; Lei n. ${ }^{\circ}$ 36/98 - Lei de Saúde Mental, 1998). A decisão sobre este tipo de medida é tomada por um juiz, com base no relatório de avaliação psiquiátrica. Os doentes, além do direito a terem um advogado de defesa nomeado, devem ser informados dos seus direitos, estar presentes nos atos processuais e serem ouvidos por um juiz. Os doentes também têm direito de votar, enviar e receber correio eletrónico e comunicar com a Comissão de Acompanhamento.

Contudo, são obrigados a cumprir o tratamento prescrito (Almeida \& Molodynski, 2016; Lei n. ${ }^{\circ} 36 / 98$ - Lei de Saúde Mental, 1998).

O internamento compulsivo de urgência ocorre se houver um perigo iminente devido a uma deterioração aguda do estado da pessoa que sofre de transtorno mental. A polícia ou as autoridades de saúde pública podem determinar que a pessoa que sofre de transtorno mental seja acompanhada à instituição mais próxima com um serviço de urgência psiquiátrico para avaliação psiquiátrica formal.

Em situações em que o atraso na avaliação do doente possa ser perigoso, qualquer policial pode proceder à escolta imediata do doente ao serviço de urgência. Nestas situações o Ministério Público local deve ser informado imediatamente. 
Quando a avaliação psiquiátrica estabelece a necessidade de internamento e o doente recusa, a instituição deve notificar a admissão do doente ao tribunal competente (mandado e relatório médico) (Almeida \& Molodynski, 2016; Lei n. ${ }^{\circ}$ 36/98 - Lei de Saúde Mental, 1998). Num prazo até 48 h após admissão do doente, o juiz deve emitir uma decisão sobre a admissão, iniciar o processo de internamento compulsivo e, consequentemente, solicitar nova avaliação psiquiátrica a realizar em 5 dias por dois psiquiatras independentes e com a possível assistência de outros profissionais da saúde mental. Com a receção desta avaliação, é definida uma data para a sessão conjunta. Nas situações em que a avaliação psiquiátrica não determina a necessidade de detenção, a pessoa deve ser imediatamente liberada. (Almeida \& Molodynski, 2016; Lei n. ${ }^{\circ}$ 36/98 - Lei de Saúde Mental, 1998).

O tratamento em ambulatório compulsivo é uma alternativa ao internamento compulsivo. Se deixar de ser seguro ou o doente não cumprir os requisitos estabelecidos pelo médico psiquiatra, o médico deve informar o tribunal e solicitar o internamento compulsivo (Almeida \& Molodynski, 2016).

O cuidar é a essência da prestação de cuidados em saúde mental. O internamento compulsivo é uma das práticas mais desafiadoras do ponto de vista ético pois toca direitos e liberdades do doente. Contudo, ainda pouco se conhece sobre a perspetiva do doente, nomeadamente através de instrumentos com evidência de validade (Gabriel, 2017; Golay, et al., 2017). Neste sentido, este trabalho tem como objetivo conhecer as perceções, atitudes e emoções dos doentes submetidos a internamento compulsivo.

\section{MÉTODOS}

Reconhecida a problemática do internamento compulsivo e dilemas inerentes, fez-se uma pesquisa do estado da arte através uma revisão integrativa da literatura, uma vez que "a revisão integrativa oferece aos profissionais de diversas áreas de atuação na saúde o acesso rápido aos resultados relevantes de pesquisas que fundamentam as condutas ou a tomada de decisão, proporcionando um saber crítico" (Mendes, Silveira, \& Galvão, 2008, p. 763).

Assim, realizou-se uma pesquisa utilizando as palavraschave: compulsory admission, involuntary hospitalization. Através da associação destes descritores, concretizou-se a pesquisa nas bases de dados EBSCO e Trip Database.
Quanto ao período de busca, este compreendeu as duas primeiras semanas de janeiro de 2018.

Na pesquisa utilizaram-se caracteres boleanos para conjugar os diferentes descritores, sendo que a expressão de pesquisa utilizada foi "compulsory admission" OR "involuntary hospitalization".

Estabeleceram-se como critérios de inclusão estudos que dessem resposta ao objetivo, publicados entre 2007 e janeiro de 2018, disponíveis em full text, de abordagem quantitativa, qualitativa e/ou revisões de literatura, nos idiomas Português, Inglês e Espanhol. Teriam de referir-se a pesquisa sobre a experiência de doentes internados compulsivamente em psiquiatria.

Deste processo (Figura 1) resultaram oito artigos que incluem uma revisão de literatura, quatro estudos observacionais, um ensaio controlado, um estudo qualitativo e um estudo de validação de escala.

Figura 1 - Processo de seleção de estudos incluídos

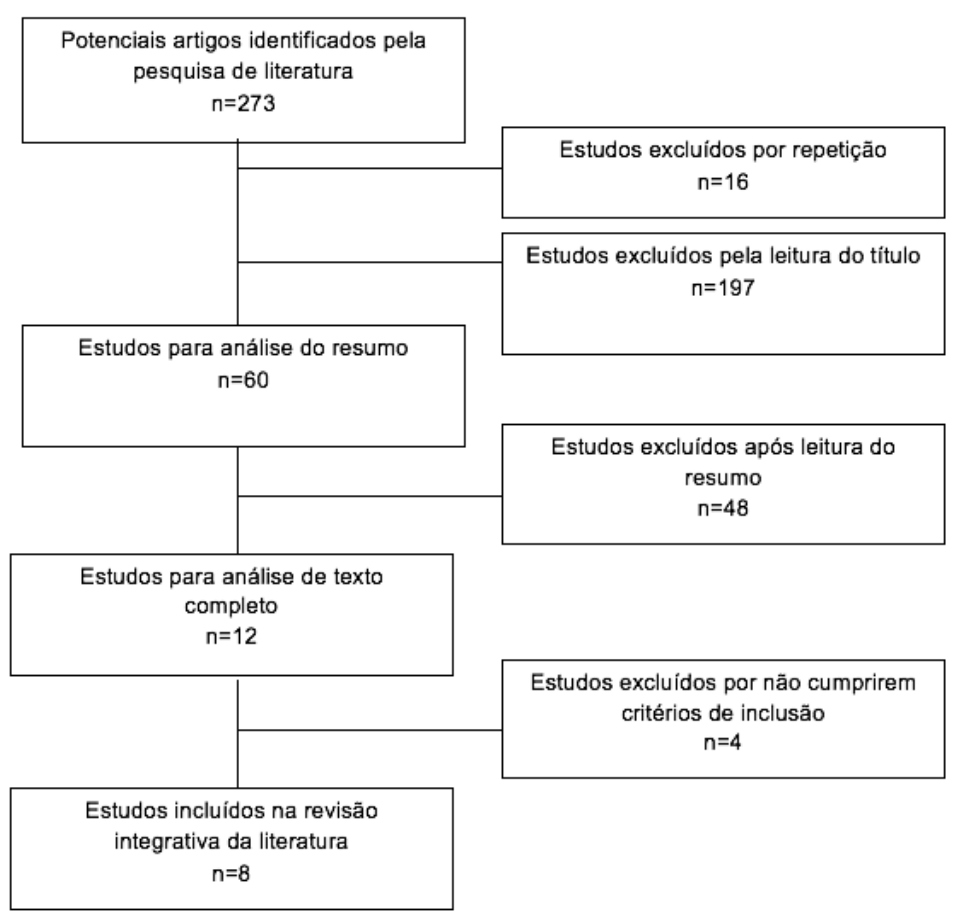

\section{RESULTADOS}

Os estudos selecionados são maioritariamente estudos qualitativos, o que pode traduzir a necessidade de conhecer melhor este fenómeno de forma exploratória. Os resultados e conclusões dos artigos são apresentados de seguida (Quadro 1). Estão organizados em aspetos negativos e positivos das perceções, atitudes e emoções dos utentes submetidos a internamento compulsivo. 
Quadro 1 - Resultados da Pesquisa

\begin{tabular}{|c|c|c|c|c|c|}
\hline No & \begin{tabular}{|l} 
Autores \\
(Ano) \\
País \\
\end{tabular} & Objetivos & Amostra & Metodologia & Resultados/Conclusões \\
\hline 1 & $\begin{array}{l}\text { Katsakou \& } \\
\text { Preibe (2007) } \\
\text { Reino Unido }\end{array}$ & $\begin{array}{l}\text { Explorar a } \\
\text { experiência de } \\
\text { internamento e } \\
\text { tratamento com- } \\
\text { pulsivo de doen- } \\
\text { tes psiquiátricos. }\end{array}$ & 5 artigos & $\begin{array}{l}\text { Revisão de } \\
\text { estudos quali- } \\
\text { tativos }\end{array}$ & $\begin{array}{l}\text { Aspetos negativos } \\
\text { Restrição da autonomia e não participação nas decisões do tratamen- } \\
\text { to: perda de liberdade, de direitos e de impotência; } \\
\text { Perceção de baixa qualidade do tratamento (serviços e profissionais): } \\
\text { ambientes não promotores do bem-estar, falta de privacidade, senti- } \\
\text { mento de rejeição e falta de disponibilidade dos profissionais; } \\
\text { Impacto emocional: desesperança, frustração, baixa autoestima inse- } \\
\text { gurança e sentimento de perda de controle. Sentimento de punição } \\
\text { por terem um problema de saúde e discriminação face a outros } \\
\text { doentes não compulsivos. Após a alta alguns continuam a sentir-se } \\
\text { desvalorizados e estigmatizadas. } \\
\text { Aspetos positivos face ao internamento compulsivo } \\
\text { Respeito pela autonomia: alguns doentes sentiram-se integrados e } \\
\text { envolvidos no seu processo de tratamento. Apesar da obrigatoriedade } \\
\text { do mesmo, percecionaram alguma liberdade e flexibilidade. } \\
\text { Tratamento e benefícios: relação positiva com profissionais que lhes } \\
\text { reduziu medos e inseguranças, relações positivas com outros doentes, } \\
\text { identificam o ambiente hospitalar como seguro e o internamento } \\
\text { compulsivo como necessário. }\end{array}$ \\
\hline 2 & $\begin{array}{l}\text { Priebe, et al. } \\
\text { (2010) } \\
\text { Reino Unido }\end{array}$ & $\begin{array}{l}\text { Explorar os } \\
\text { pontos de vista } \\
\text { dos doentes após } \\
\text { internamento } \\
\text { involuntário em } \\
\text { diferentes países } \\
\text { europeus. }\end{array}$ & $n=2326$ & $\begin{array}{l}\text { Estudo ob- } \\
\text { servacional } \\
\text { prospetivo }\end{array}$ & $\begin{array}{l}\text { Aspetos negativos } \\
\text { Aqueles que sofrem de esquizofrenia tiveram opiniões mais negati- } \\
\text { vas. } \\
\text { Aspetos positivos } \\
\text { Um mês após o internamento compulsivo } 55 \% \text { concordam retrospeti- } \\
\text { vamente com a adequação da admissão compulsiva e após três meses, } \\
63 \% \text {. Há variações significativas entre os países. Género, situação de } \\
\text { vida e diagnóstico foram identificados como fatores que influenciam } \\
\text { a opinião dos doentes. }\end{array}$ \\
\hline 3 & $\begin{array}{l}\text { Sibitz, et al. } \\
\text { (2011) } \\
\text { Reino Unido }\end{array}$ & $\begin{array}{l}\text { Estabelecer uma } \\
\text { tipologia de } \\
\text { perspetivas de } \\
\text { coerção. }\end{array}$ & $\mathrm{n}=15$ & $\begin{array}{l}\text { Estudo quali- } \\
\text { tativo }\end{array}$ & $\begin{array}{l}\text { Aspetos negativos } \\
\text { As pessoas descreveram sentimentos de humilhação, desrespeito } \\
\text { e desamparo. Identificaram a experiência como algo exagerado e } \\
\text { desnecessário. O impacto negativo da experiência: diminuição da } \\
\text { autoestima, sentimento de impotência e falta de controlo, reserva } \\
\text { e defesa nas relações sociais, perceção de que as medidas coercivas } \\
\text { promoveram o prolongamento do internamento, efeitos negativos no } \\
\text { desempenho de papéis sociais e recuperação pobre. A maioria dos } \\
\text { participantes questionou a forma como foram aplicadas as medidas } \\
\text { coercivas. } \\
\text { Aspetos positivos } \\
\text { Outros identificaram como algo necessário quando confrontados } \\
\text { com o perigo para si ou para os outros. Impacto positivo: ajudou a } \\
\text { encarar a doença mais seriamente, promoveu a valorização de uma } \\
\text { situação estável e a ser mais calmo quando confrontado com a adver- } \\
\text { sidade. }\end{array}$ \\
\hline 4 & $\begin{array}{l}\text { Rusch, et al. } \\
\text { (2014) } \\
\text { Suiça }\end{array}$ & $\begin{array}{l}\text { Conhecer } \\
\text { reações emocio- } \\
\text { nais e cognitivas } \\
\text { relacionadas ao } \\
\text { estigma com } \\
\text { internamento } \\
\text { involuntário e } \\
\text { seu impacto em } \\
\text { pessoas com } \\
\text { doença mental. }\end{array}$ & $\mathrm{n}=186$ & $\begin{array}{l}\text { Ensaio con- } \\
\text { trolado }\end{array}$ & $\begin{array}{l}\text { Aspetos negativos } \\
\text { Reações ao internamento involuntário: vergonha, autodesprezo, baixa } \\
\text { autoestima, que podem levar ao auto estigma, ao empowerment } \\
\text { reduzido e à baixa qualidade de vida. } \\
\text { Aspetos positivos } \\
\text { As intervenções para reduzir os efeitos negativos das admissões } \\
\text { obrigatórias devem abordar reações emocionais, empowerment e } \\
\text { capacitação. }\end{array}$ \\
\hline
\end{tabular}




\begin{tabular}{|c|c|c|c|c|c|}
\hline 5 & $\begin{array}{l}\text { Gabriel } \\
(2017) \\
\text { Canadá }\end{array}$ & $\begin{array}{l}\text { Construir } \\
\text { e avaliar as } \\
\text { propriedades } \\
\text { psicométricas de } \\
\text { um instrumento } \\
\text { para avaliar a } \\
\text { atitude dos paci- } \\
\text { entes em relação } \\
\text { ao internamento } \\
\text { involuntário }\end{array}$ & $\mathrm{N}=80$ & $\begin{array}{l}\text { Revisão } \\
\text { abrangente da } \\
\text { literatura } \\
\text { Validação de } \\
\text { escala }\end{array}$ & $\begin{array}{l}\text { Aspetos negativos } \\
\text { Identificaram sentimento de violação de direitos legais e autonomia: } \\
\text { ameaça à independência, humilhação e estigmatização por parte de } \\
\text { outros. } \\
\text { Aspetos positivos } \\
\text { Atitudes positivas: sentimento de respeito, reconhecimento da neces- } \\
\text { sidade do internamento. Apesar da coerção ser mais frequentemente } \\
\text { relatada pelos doentes com admissão involuntária, esta também foi } \\
\text { identificada pelos doentes admitidos voluntariamente. Atitudes nega- } \\
\text { tivas face ao internamento compulsivo mudam com o tempo. } \\
\text { Ambivalência apesar do reconhecimento da necessidade do interna- } \\
\text { mento, sentiram que a admissão poderia ter sido voluntária e sem } \\
\text { pressão; }\end{array}$ \\
\hline 6 & $\begin{array}{l}\text { Jaeger, et al. } \\
(2013) \\
\text { Alemanha }\end{array}$ & $\begin{array}{l}\text { Examinar a } \\
\text { influência a } \\
\text { longo prazo do } \\
\text { internamento } \\
\text { compulsivo } \\
\text { sobre a adesão } \\
\text { à medicação, o } \\
\text { envolvimento no } \\
\text { tratamento e na } \\
\text { coerção perce- } \\
\text { bida. }\end{array}$ & $\begin{array}{l}290 \text { in- } \\
\text { ternados } \\
\text { voluntar- } \\
\text { iamente } \\
\text { e } 84 \\
\text { pacientes } \\
\text { hospital- } \\
\text { izados } \\
\text { invol- } \\
\text { untari- } \\
\text { amente } \\
\end{array}$ & $\begin{array}{l}\text { Estudo mul- } \\
\text { ticultural de } \\
\text { observação } \\
\text { naturalista }\end{array}$ & $\begin{array}{l}\text { Aspetos negativos } \\
\text { A hospitalização involuntária não parece prejudicar o envolvimento } \\
\text { futuro com o tratamento de doentes com esquizofrenia, mas pacien- } \\
\text { tes anteriormente hospitalizados involuntariamente mostram-se mais } \\
\text { sensíveis à coerção subjetiva ou real e mais vulneráveis à não adesão } \\
\text { à medicação. Por isso, o risco de hospitalização involuntária futura } \\
\text { pode ser aumentado. }\end{array}$ \\
\hline 7 & $\begin{array}{l}\text { O’Donogue, } \\
\text { et al. (2011) } \\
\text { Reino Unido }\end{array}$ & $\begin{array}{l}\text { Investigar a } \\
\text { perceção das } \\
\text { pessoas sobre a } \\
\text { necessidade de } \\
\text { sua admissão } \\
\text { involuntária no } \\
\text { ano seguinte ao } \\
\text { da alta e taxas de } \\
\text { readmissão para } \\
\text { o hospital e a } \\
\text { influência do in- } \\
\text { sight e do estilo } \\
\text { de recuperação. }\end{array}$ & $\mathrm{n}=81$ & $\begin{array}{l}\text { Estudo ob- } \\
\text { servacional } \\
\text { retrospetivo }\end{array}$ & $\begin{array}{l}\text { Aspetos negativos } \\
\text { Dos que mudaram seus pontos de vista, a maioria refletiu negativa- } \\
\text { mente sobre sua admissão involuntária. } \\
\text { Aspetos positivos } \\
\text { Na primeira entrevista } 72 \% \text { relataram que sua admissão involuntária } \\
\text { tinha sido necessária, um ano após a alta apenas } 60 \% \text {. }\end{array}$ \\
\hline 8 & $\begin{array}{l}\text { Smith, et al. } \\
(2014) \\
\text { Irlanda }\end{array}$ & $\begin{array}{l}\text { Avaliar a sat- } \\
\text { isfação com os } \\
\text { serviços após } \\
\text { a admissão } \\
\text { psiquiátrica e } \\
\text { explorar sua re- } \\
\text { lação com uma } \\
\text { série de fatores } \\
\text { clínicos e de } \\
\text { serviços. }\end{array}$ & $\mathrm{n}=161$ & $\begin{array}{l}\text { Estudo de } \\
\text { observação } \\
\text { multicêntrico }\end{array}$ & $\begin{array}{l}\text { Aspetos negativos } \\
\text { Menor satisfação relaciona-se com coerção física, administração de } \\
\text { medicação sem consentimento, menores níveis de justiça processual, } \\
\text { medidas de isolamento e uso de substâncias. } \\
\text { Aspetos positivos } \\
\text { O nível geral de satisfação com os serviços de internamento de } \\
\text { psiquiatria foi bom. Uma melhor relação terapêutica, maior insight e } \\
\text { maior funcionalidade foram associadas a maiores níveis de satisfação } \\
\text { do tratamento. }\end{array}$ \\
\hline
\end{tabular}

\section{DISCUSSÃO}

De acordo com os artigos encontrados, pode-se constatar que a pesquisa atual surge maioritariamente sob a forma qualitativa e de origem europeia. Os resultados dos estudos revelam que o internamento compulsivo é um processo complexo e pode ter impacto nas vidas dos doentes (Katsakou \& Preibe, 2007). Por vezes envolve práticas de coerção física ou psicológica e, neste contexto, os doentes são confrontados com as regras externas impostas por profissionais (Katsakou \& Preibe, 2007). Estas vivências são percecionadas e sentidas de forma negativa, positiva e ambivalente, identificando práticas que necessitam de ser melhoradas (Quadro 1). 


\section{Perceções, Atitudes e Emoções Negativas}

As perceções, atitudes e emoções negativas identificadas pelos doentes face ao internamento compulsivo são: a perceção de restrição de autonomia, ameaça à independência, a não participação nas decisões do tratamento, a perda de liberdade, de direitos, do poder de decidir por eles mesmos e o sentimento de impotência (Katsakou \& Preibe, 2007; Gabriel, 2017). Descreveram ainda: uma perceção de baixa qualidade do tratamento (serviços e profissionais), nomeadamente, ambientes não promotores do bem-estar, falta de privacidade, sentimento de rejeição pela sensação de falta de abertura/ disponibilidade dos profissionais e medo dos outros doentes (Katsakou \& Preibe, 2007).

Quando as pessoas sentem que não têm voz no tratamento que recebem e são tratadas desrespeitosamente pelos profissionais, a sua autoconfiança é automaticamente afetada e eles sentem que são seres humanos inferiores, com menos valor do que outras pessoas. Estes aspetos têm um impacto emocional negativo, nomeadamente sentimentos de desesperança, frustração, baixa autoestima, vergonha, insegurança e sentimento de perda de controle sobre suas vidas (Katsakou \& Preibe, 2007; Rusch, et al., 2014; Gabriel, 2017). Os doentes descreveram situações em que se sentiram humilhados, desrespeitados, desemparados, sozinhos e alguns acreditam que as medidas coercivas levaram a um prolongamento do internamento (Sibitz, et al., 2011). Sentem-se punidos por terem um problema de saúde e discriminados face a outros doentes não compulsivos. Após a alta, alguns continuaram a sentir-se desvalorizados e estigmatizados (Katsakou \& Preibe, 2007), apresentaram auto estigma, empowerment reduzido, baixa qualidade de vida (Rusch, et al., 2014), maior reserva nas relações sociais, efeitos negativos no desempenho de papéis sociais e uma perceção negativa que influenciou uma recuperação pobre (Sibitz, et al., 2011).

De acordo com um estudo realizado por Jaeger et al. (2013), a hospitalização involuntária não parece prejudicar o envolvimento futuro no tratamento dos doentes com esquizofrenia, no entanto, doentes anteriormente hospitalizados involuntariamente mostram-se mais sensíveis à coerção subjetiva ou real e posteriormente aderem menos à medicação. Por isso, o risco de hospitalização involuntária futura pode ser aumentado (Jaeger, et al., 2013). Neste sentido, a satisfação dos doentes é um indicador a ter em consideração.

De uma forma geral os doentes internados em psiquiatria têm uma boa satisfação com os serviços.
No entanto, verifica-se que os doentes admitidos involuntariamente, que sofreram coerção física, que foram colocados em isolamento, aos quais foi administrada medicação contra a sua vontade, que sentiram menores níveis de justiça processual, mostraram-se menos satisfeitos.

Há, ainda, fatores externos ao internamento que também podem influenciar o nível de satisfação e o uso de substâncias foi identificado como tal (Smith, et al., 2014). As atitudes negativas face ao internamento compulsivo tendem a mudar com o tempo. Num estudo realizado em onze países europeus foram entrevistados 1809 doentes psiquiátricos internados compulsivamente um mês após admissão e 1613 foram entrevistados três meses depois. Os resultados revelaram que um mês após o internamento compulsivo 55\% concorda retrospetivamente com a adequação da admissão compulsiva e após três meses, 63\% (Priebe, et al., 2010). No entanto, um outro estudo realizado em um hospital psiquiátrico em Dublin (Hospital St John of God) com 81 doentes internados compulsivamente, dos quais 68 foram reentrevistados um ano depois do internamento, revelou que a maioria refletiu negativamente sobre sua admissão involuntária: desses, 33\% acreditavam anteriormente na necessidade do internamento compulsivo, mudando de opinião um ano depois (O'Donogue, et al., 2011).

Para além de haver variações significativas entre países diferentes, fatores como gênero, situação de vida e diagnóstico, influenciam as vivências do internamento compulsivo (Priebe, et al., 2010).

\section{Perceções, Atitudes e Emoções Positivas}

Nos estudos encontrados, para além de perceções, atitudes e emoções negativas, os doentes internados compulsivamente também identificaram perceções, atitudes e emoções positivas na sua experiência (quadro 1). Alguns doentes sentiram-se bem integrados e envolvidos no seu processo terapêutico, valorizaram o facto de lhes ser dada informação sobre os seus direitos e tratamento e, apesar da obrigatoriedade do internamento compulsivo, percecionaram alguma liberdade e flexibilidade (Katsakou \& Preibe, 2007). Referiram, ainda, que uma relação positiva com os profissionais lhes reduziu medos e inseguranças, identificaram relações positivas com outros doentes, sentiram o ambiente hospitalar como seguro, e reconheceram o internamento compulsivo como necessário e com resultados benéficos (Katsakou \& Preibe, 2007). 
Descreveram que o internamento compulsivo os ajudou a encarar a doença mais seriamente, promoveu a valorização de uma situação estável, contribuiu para valorizar a necessidade de aproveitar a vida cotidiana e ser mais calmo quando confrontado com a adversidade (Sibitz, et al., 2011).

Melhores níveis de satisfação com o tratamento em internamento compulsivo estão relacionados com uma melhor relação terapêutica. Também foram identificados fatores associados a maiores níveis de satisfação do tratamento que não estão propriamente relacionados com o internamento, mas podem ser trabalhados nesse âmbito, que são o maior insight e maior funcionalidade (Smith, et al., 2014).

\section{Perceções, Atitudes e Emoções Ambivalentes}

Alguns doentes mostraram-se ambivalentes, apesar do reconhecimento de que houve necessidade de tratamento e de que a admissão evitou maiores danos, sentiram que a admissão poderia ter sido realizada de forma voluntária e sem pressão (Gabriel, 2017).

Muitos dos doentes, participantes nos estudos encontrados, questionaram a forma como foram aplicadas as medidas coercivas identificando-as como práticas que precisam ser substancialmente melhoradas para contrariar possíveis efeitos adversos a longo prazo (Sibitz, et al., 2011).

\section{IMPLICAÇÕES PARA A PRÁTICA CLÍNICA}

Os achados destes estudos poderão ter implicações significativas para os cuidados em contexto de internamento compulsivo. Quando os doentes sentem que são respeitados, que os profissionais estão genuinamente interessados e envolvidos no seu tratamento, quando lhes é oferecido algum grau de participação e autonomia no seu tratamento, estas medidas são vistas com um impacto menos negativo na sua autoestima e é-lhes mais fácil aceitar o internamento compulsivo (Katsakou \& Preibe, 2007).

Assim sendo, é necessário melhorar a participação das pessoas no seu processo de internamento, bem como, a perceção que têm dessa participação, mesmo que admitidas involuntariamente; fornecer informação sobre o tratamento e envolver o doente na discussão de possíveis alternativas; melhorar a interação entre doentes e profissionais e otimizar a relação com os outros doentes; e promover um ambiente seguro (Katsakou \& Preibe, 2007).
É também muito importante que os serviços/cuidados de saúde salvaguardem, apesar da necessidade de tratar compulsivamente alguns doentes, o objetivo a longo prazo de promoção de autonomia e independência que pode estar em causa nos doentes que têm alta com sentimentos de inferioridade e baixo empowerment (Katsakou \& Preibe, 2007). As intervenções para reduzir os efeitos negativos das admissões obrigatórias devem abordar reações emocionais, empowerment e capacitação (Rusch, et al., 2014).

\section{CONCLUSÃO}

Em geral, as pessoas que padecem de transtorno mental são particularmente sensíveis a qualquer intrusão na sua independência e privacidade, e as medidas compulsivas e coercivas são vistas como um ataque à sua autonomia e liberdade.

É importante compreender como a exposição a internamentos compulsivos, que muitas vezes envolvem medidas coercivas, pode fazer com que os doentes se sintam expostos e vulneráveis.

As restrições da autonomia são provavelmente inevitáveis quando as pessoas são tratadas contra a sua vontade (Katsakou \& Preibe, 2007). Nesta revisão de literatura foram identificadas perceções, atitudes e emoções positivas e negativas face a experiências vivenciadas por doentes sujeitos a internamento compulsivo e ainda ambivalência, o que nos remete para a necessidade de melhoria de práticas nos cuidados prestados. Foi também possível perceber que estas vivências quando percecionadas como negativas podem ter consequências a longo prazo, nomeadamente baixa autoestima, vergonha, insegurança e sentimento de perda de controlo sobre suas vidas, empowerment reduzido e baixa qualidade de vida, maior reserva nas relações sociais, efeitos negativos no desempenho de papéis sociais e uma recuperação pobre.

Foram também referenciadas perceções, atitudes e emoções positivas face ao internamento compulsivo, nomeadamente o sentimento de que esta experiência os ajudou a encarar a doença mais seriamente, promoveu a valorização de uma situação estável, maior valorização da necessidade de aproveitar a vida cotidiana e ser mais calmo quando confrontado com a adversidade. 
As perceções, atitudes e emoções negativas, positivas e a ambivalência, associadas à identificação da necessidade de melhoria dos cuidados prestados, ajudaram a identificar práticas que melhoram a experiência do doente sujeito a internamento compulsivo, nomeadamente intervenções que abordem reações emocionais, empoderamento e capacitação, melhoria da relação doente-profissional, envolvimento do doente no seu tratamento, informação sobre direitos e tratamento com o objetivo a longo prazo de promoção de autonomia e independência.

A experiência de internamento compulsivo é algo complexo que sofre influencia de diversos fatores e alguns deles não necessariamente relacionados com o internamento em si. Sugere-se a realização de estudos que identifiquem melhor a influência destes fatores nas vivências do internamento compulsivo. Face à prevalência de estudos qualitativos na área, sugere-se, ainda o desenvolvimento de mais estudos quantitativos sobre o tema. Como não foi encontrada evidência sobre a temática em Portugal, aponta-se a necessidade de conhecer as vivências dos doentes sujeitos a internamentos compulsivos no nosso país.

\section{REFERÊNCIAS BIBLIOGRÁFICAS}

Almeida, T. \& Molodynski, A. (2016). Compulsory admission and involuntary treatment in Portugal: Mental Health Law Profile. British Journal of Psychiatry, 13 (1), 17-19. Disponível em: https://www.ncbi.nlm.nih.gov/ pmc/articles/PMC5618893/

Caldas de Almeida, J. M. (2009). Portuguese National Mental Health Plan (2007-2016) executive summary. Mental Health in Family Medicine, 6 (4), 233-244. Disponível em: https://www.ncbi.nlm.nih.gov/pmc/articles/PMC2873880/

European Commission. (2002). Compulsory Admission and Involuntary Treatment of Mentally Ill Patients - Legislation and Practice in EU-Member States. Projeto de pesquisa, Health \& Consumer Protection Directorate-General, Alemanha.

Gabriel, A. (2017). Development of an instrument to measure patients' attitudes towards involuntary hospitalization. World Journal of Psychiatry, 7 (2), 89-97. Doi: 10.5498/wjp.v7.i2.89
Golay, P., Semlali, I., Beuchat, H., Pomini, V., Silva, B., Loutrel, L., Thonney, J., Gallo, S., Morandi, S., Bonsack, C. (2017). Perceived coercion in psychiatric hospital admission: validation of the Frenchlanguage version of the MacArthur Admission Experience Survey. BioMedCentral Psychiatry, 17 (357),1-8. Doi: 10.1186/s12888017-1519-4

Jaeger, S., Pfiffner, C., Weiser, P., Langle, G., Croissant, D., Schepp, W., Kilian, R., Becker, T., Eschweiler, G., Steinert, T. (2013). Long-term effects of involuntary hospitalization on medication adherence, treatment engagement and perception of coercion. Social Psychiatry and Psychiatric Epidemiology, 48 (11), 1787-1796. Doi: $10.1007 / \mathrm{s} 00127-013-0687-\mathrm{x}$

Katsakou, C. \& Preibe, S. (2007). Patient's experiences of involuntary hospital admission and treatment: Areview of qualitative studies. Epidemiologia e Psichiatria Sociale, 16 (2), 172-178.

Lei n. ${ }^{\circ}$ 36/98 - Lei de Saúde Mental. (24 de Julho de 1998). Diário da República n. ${ }^{\circ}$ 169/1998, de 1998-0724, Série I-A. Portugal/Lisboa.

Mendes, K., Silveira, R., \& Galvão, C. (2008). Revisão Integrativa: Método de Pesquisa para a Incorporação de Evidências na Saúde e na Enfermagem. Texto Contexto Enfermagem, 17 (4), 758-64. Doi: 10.1590/S010407072008000400018

Myklebust, L., Sørgaard, K., Røtvold, K., \& Wynn, R. (2012). Factors of importance to involuntary admission. Informa Healthcare, 66 (3), 178-182. Doi: $10.3109 / 08039488.2011 .611252$

O’Donogue, B., Lyne, J., Hill, M., O’Rourke, L., Daly, S., Larkin, C., Feeney, L., O'Callaghan, E. (2011). Perceptions of involuntary admission and risk of subsequent readmission at one-year follow-up: The influence of insight and recovery style. Journal of Mental Health, 20 (3), 249-259. Doi: 10.3109/09638237.2011.562263

Priebe, S., Katsakou, C., Glockner, M., Dembinskas, A., Fiorillo, A., Karastergiou, A., Kiejna, A., Kjellin, L., Nawka, P., Onchev, G., Raboch, J., Schuetzwoh, M., Solomon, Z., Torres-Gonzalez, F., Wang, D., Kallert, T. (2010). Patients' views of involuntary hospital admission after 1 and 3 months: prospective study in $11 \mathrm{Eu}$ ropean countries. The British Journal of Psychiatry, 196 (3),179-185. Doi: 10.1192/bjp.bp.109.068916 
Rusch, N., Muller, M., Lay, B., Corrigan, P., Zahn, R., Schonenberger, T., Bleiker, M., Lengler, S., Blank, C., Rossler, W. (2014). Emotional reactions to involuntary psychiatric hospitalization and stigma-related stress among people with mental illness. European Archives of Psychiatry and Clinical Neuroscience, 264 (1), 3543. Doi: $10.1007 / \mathrm{s} 00406-013-0412-5$

Sibitz, I., Scheutz, A., Lakeman, R., Schrank, B., Schaffer, M., \& Amering, M. (2011). Impact of coercive measures on life stories: qualitative study. The British Journal of Psychiatry, 199 (3), 239-244. Doi: 10.1192/bjp. bp. 110.087841

Smith, D., Roche, E., O’Loughlin, K., Brennan, D., Madigan, K., Lyne, J., Feeney, L., O’Donoghue, B. (2014). Satisfaction with services following voluntary and involuntary admission. Journal of Ment Health, 23 (1), 38-45. Doi: 10.3109/09638237.2013.841864
Xavier, M. (2002). Compulsory Admission and Involuntary Treatment in the EU - Portugal . In E. C.-H. Directorate-General, Compulsory Admission and Involuntary Treatment of Mentally Ill Patients - Legislation and Practice in EU-Member States (pp. 123-130). Alemanha.

Xavier, M. \& Carvalho, A. (s.d.). Internamento Compulsivo em Portugal - Contexto e Procedimentos. Lisboa: DGS-Direção Geral de Saúde.

Zinkler, M. \& Priebe, S. (2002). Detention of the mentally ill in Europe - a review. Acta Psychiatrica Scandinavica, 106 (1), 3-8. Disponível em: https://www.ncbi. nlm.nih.gov/pubmed/12100342

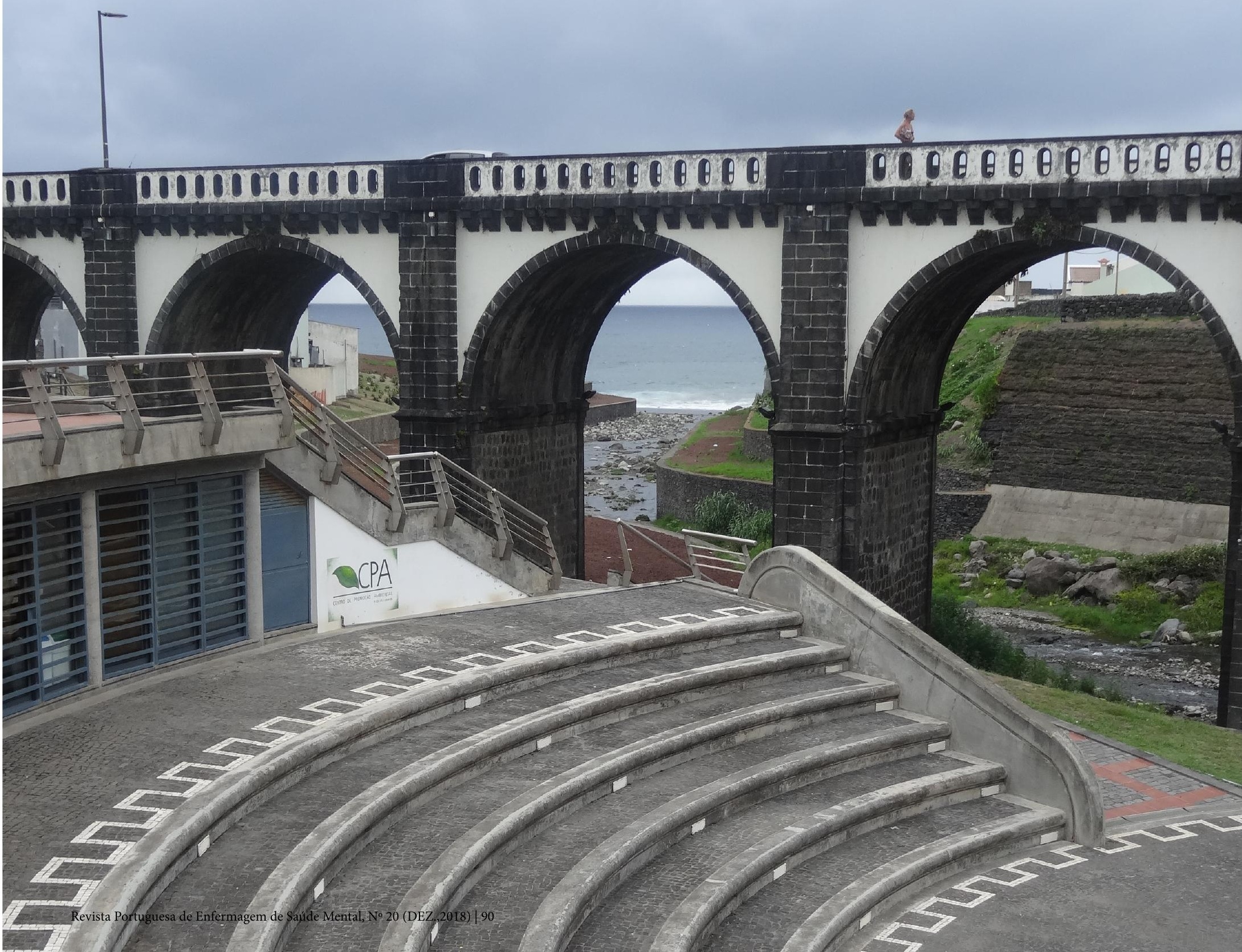

